\title{
THE DISCOVERY OF $\gamma$-RAY EMISSION FROM THE BLAZAR RGB J0710+591
}

V. A. Acciari ${ }^{1}$, E. Aliu ${ }^{2}$, T. Arlen ${ }^{3}$, T. Aune ${ }^{4}$, M. Bautista ${ }^{5}$, M. Beilicke ${ }^{6}$, W. Benbow ${ }^{1}$, M. Böttcher ${ }^{7}$, D. Boltuch ${ }^{8}$,

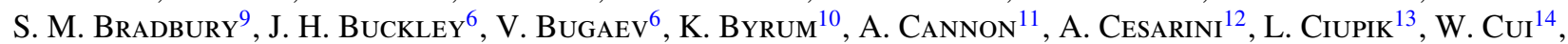
R. Dickherber ${ }^{6}$, C. Duke ${ }^{15}$, A. Falcone ${ }^{16}$, J. P. Finley ${ }^{14}$, G. Finnegan ${ }^{17}$, L. Fortson ${ }^{13}$, A. Furniss ${ }^{4}$, N. Galante ${ }^{1}$, D. Gall ${ }^{14}$, K. Gibbs ${ }^{1}$, G. H. Gillanders ${ }^{12}$, S. Godambe ${ }^{17}$, J. Grube ${ }^{11}$, R. Guenette ${ }^{5}$, G. GyuK ${ }^{13}$, D. Hanna ${ }^{5}$, J. Holder ${ }^{8}$, C. M. HuI ${ }^{17}$, T. B. HumenSKY ${ }^{18}$, A. Imran ${ }^{19}$, P. KAARET ${ }^{20}$, N. Karlsson ${ }^{13}$, M. KerTZMAN ${ }^{21}$, D. KIEDA ${ }^{17}$, A. KonOPELKO ${ }^{22}$, H. KrawcZynski ${ }^{6}$, F. Krennrich ${ }^{19}$, M. J. Lang ${ }^{12}$, A. Lamerato ${ }^{7}$, S. LeBohec ${ }^{17}$, G. Maier ${ }^{5,81}$, S. McArthur ${ }^{6}$, A. McCanN ${ }^{5}$, M. McCutcheon ${ }^{5}$, P. Moriarty ${ }^{23}$, R. Mukherjee ${ }^{2}$, R. A. $\mathrm{Ong}^{3}$, A. N. Otte ${ }^{4}$, D. Pandel ${ }^{20}$, J. S. Perkins ${ }^{1}$, D. Petri ${ }^{24}$,

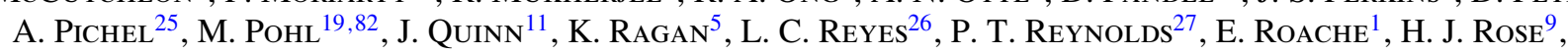
P. Roustazadeh ${ }^{7}$, M. Schroedter ${ }^{19}$, G. H. Sembroski ${ }^{14}$, G. Demet Senturk ${ }^{28}$, A. W. Smith ${ }^{10}$, D. Steele ${ }^{13,83}$, S. P. Swordy ${ }^{18}$,

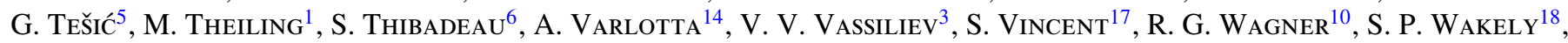
J. E. Ward ${ }^{11}$, T. C. Weekes ${ }^{1}$, A. Weinstein ${ }^{3}$, T. Weisgarber ${ }^{18}$, D. A. Williams ${ }^{4}$, S. Wissel $^{18}$, M. Wood $^{3}$, B. Zitzer ${ }^{14}$, M. ACKermanN ${ }^{29}$, M. Ajello ${ }^{29}$, E. Antolini ${ }^{30,31}$, L. Baldini ${ }^{32}$, J. Ballet ${ }^{33}$, G. Barbiellini ${ }^{34,35}$, D. Bastieri ${ }^{36,37}$,

K. Bechtol ${ }^{29}$, R. Bellazzini ${ }^{32}$, B. Berenit ${ }^{29}$, R. D. BlandFord ${ }^{29}$, E. D. Bloom ${ }^{29}$, E. Bonamente ${ }^{30,31}$, A. W. Borgland ${ }^{29}$, A. Bouvier ${ }^{29}$, J. Bregeon ${ }^{32}$, M. Brigida ${ }^{38,39}$, P. Bruel ${ }^{40}$, R. Buehler ${ }^{29}$, S. Buson ${ }^{36}$, G. A. Caliandro ${ }^{41}$, R. A. Cameron ${ }^{29}$, P. A. Caraver ${ }^{42}$, S. Carrigan ${ }^{37}$, J. M. Casandjian ${ }^{33}$, E. Cavazzuti ${ }^{43}$, C. Cecchi ${ }^{30,31}$, Ö. Çelik ${ }^{44,45,46}$, E. Charles ${ }^{29}$, A. CheKhtman ${ }^{47,48}$, C. C. Cheung ${ }^{47,49}$, J. Chiang ${ }^{29}$, S. Ciprini $^{31}$, R. Claus ${ }^{29}$, J. Cohen-Tanugi ${ }^{50}$, J. Conrad ${ }^{51,52,84}$, C. D. Dermer ${ }^{47}$, F. De Palma ${ }^{38,39}$, E. do Couto e Silva ${ }^{29}$, P. S. Drell ${ }^{29}$, R. Dubois ${ }^{29}$, D. Dumora ${ }^{53,54}$, C. Farnier ${ }^{50}$,

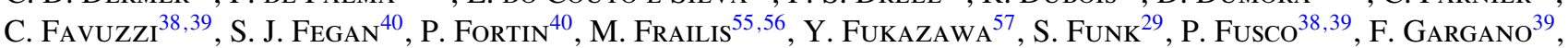
D. Gasparrini ${ }^{43}$, N. Gehrels ${ }^{44}$, S. Germani ${ }^{30,31}$, B. Giebels ${ }^{40}$, N. Giglietto ${ }^{38,39}$, F. Giordano $^{38,39}$, M. Giroletti ${ }^{58}$, T. Glanzman ${ }^{29}$, G. Godfrey ${ }^{29}$, I. A. Grenier ${ }^{33}$, J. E. Grove ${ }^{47}$, S. Guiriec ${ }^{59}$, E. Hays ${ }^{44}$, D. Horan ${ }^{40}$, R. E. Hughes ${ }^{60}$,

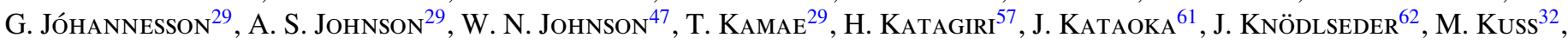

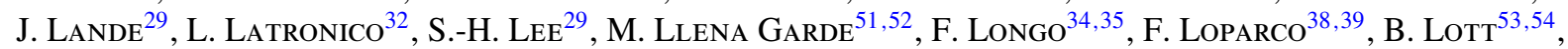
M. N. Lovellette ${ }^{47}$, P. Lubrano ${ }^{30,31}$, A. Makeev ${ }^{47,48}$, M. N. Mazziotta ${ }^{39}$, P. F. Michelson ${ }^{29}$, W. Mitthumisiri ${ }^{29}$, T. Mizuno ${ }^{57}$, A. A. Moiseev ${ }^{45,63}$, C. Monte ${ }^{38,39}$, M. E. MonZani ${ }^{29}$, A. Morselli ${ }^{64}$, I. V. Moskalenko ${ }^{29}$, S. Murgia ${ }^{29}$,

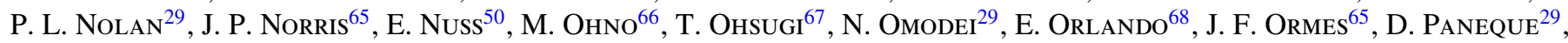

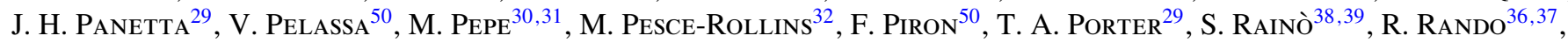

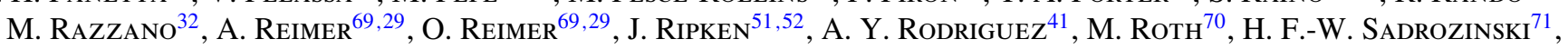
D. Sanchez ${ }^{40}$, A. Sander ${ }^{60}$, J. D. Scargle ${ }^{72}$, C. Sgrò ${ }^{32}$, E. J. Siskind ${ }^{73}$, P. D. Smith ${ }^{60}$, G. SPandre ${ }^{32}$, P. Spinelli ${ }^{38,39}$,

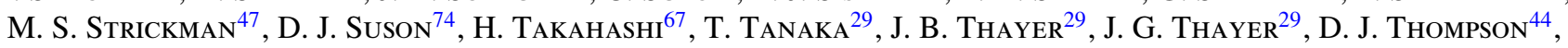
L. Tibaldo ${ }^{33,36,37,85}$, D. F. Torres ${ }^{41,75}$, G. Tosti ${ }^{30,31}$, A. Tramacere ${ }^{29,76,77}$, T. L. Usher ${ }^{29}$, V. VAsileiou ${ }^{45,46}$, N. VilcheZ ${ }^{62}$,

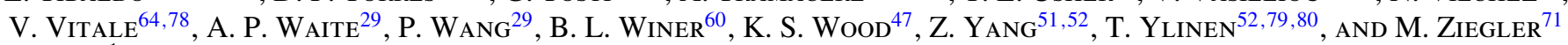

${ }^{1}$ Fred Lawrence Whipple Observatory, Harvard-Smithsonian Center for Astrophysics, Amado, AZ 85645, USA; jperkins@ @ cfa.harvard.edu

${ }^{2}$ Department of Physics and Astronomy, Barnard College, Columbia University, NY 10027, USA

${ }^{3}$ Department of Physics and Astronomy, University of California, Los Angeles, CA 90095, USA

${ }^{4}$ Santa Cruz Institute for Particle Physics and Department of Physics, University of California, Santa Cruz, CA 95064, USA

${ }^{5}$ Physics Department, McGill University, Montreal, QC H3A 2T8, Canada

${ }^{6}$ Department of Physics, Washington University, St. Louis, MO 63130, USA

${ }^{7}$ Department of Physics and Astronomy, Astrophysical Institute, Ohio University, Athens, OH 45701, USA

${ }^{8}$ Department of Physics and Astronomy and the Bartol Research Institute, University of Delaware, Newark, DE 19716, USA

${ }^{9}$ School of Physics and Astronomy, University of Leeds, Leeds, LS2 9JT, UK

${ }^{10}$ Argonne National Laboratory, 9700 S. Cass Avenue, Argonne, IL 60439, USA

${ }^{11}$ School of Physics, University College Dublin, Belfield, Dublin 4, Republic of Ireland

${ }^{12}$ School of Physics, National University of Ireland Galway, University Road, Galway, Republic of Ireland

${ }^{13}$ Astronomy Department, Adler Planetarium and Astronomy Museum, Chicago, IL 60605, USA

${ }_{15}^{14}$ Department of Physics, Purdue University, West Lafayette, IN 47907, USA

${ }^{15}$ Department of Physics, Grinnell College, Grinnell, IA 50112-1690, USA

${ }^{16}$ Department of Astronomy and Astrophysics, 525 Davey Lab, Pennsylvania State University, University Park, PA 16802, USA

${ }^{17}$ Department of Physics and Astronomy, University of Utah, Salt Lake City, UT 84112, USA

${ }^{18}$ Enrico Fermi Institute, University of Chicago, Chicago, IL 60637, USA

${ }^{19}$ Department of Physics and Astronomy, Iowa State University, Ames, IA 50011, USA

${ }^{20}$ Department of Physics and Astronomy, University of Iowa, Van Allen Hall, Iowa City, IA 52242, USA

${ }^{21}$ Department of Physics and Astronomy, DePauw University, Greencastle, IN 46135-0037, USA

${ }^{22}$ Department of Physics, Pittsburg State University, 1701 South Broadway, Pittsburg, KS 66762, USA

${ }^{23}$ Department of Life and Physical Sciences, Galway-Mayo Institute of Technology, Dublin Road, Galway, Republic of Ireland

${ }^{24}$ European Southern Observatory, Karl-Schwarzschild-Strasse 2, 85748 Garching, Germany

${ }^{25}$ Instituto de Astronomia y Fisica del Espacio, Casilla de Correo 67-Sucursal 28, (C1428ZAA) Ciudad Autónoma de Buenos Aires, Argentina

${ }^{26}$ Kavli Institute for Cosmological Physics, University of Chicago, Chicago, IL 60637, USA

${ }^{27}$ Department of Applied Physics and Instrumentation, Cork Institute of Technology, Bishopstown, Cork, Republic of Ireland

${ }^{28}$ Columbia Astrophysics Laboratory, Columbia University, New York, NY 10027, USA

${ }^{29}$ W. W. Hansen Experimental Physics Laboratory, Kavli Institute for Particle Astrophysics and Cosmology, Department of Physics and SLAC National Accelerator Laboratory, Stanford University, Stanford, CA 94305, USA

${ }^{30}$ Istituto Nazionale di Fisica Nucleare, Sezione di Perugia, I-06123 Perugia, Italy 
${ }^{31}$ Dipartimento di Fisica, Università degli Studi di Perugia, I-06123 Perugia, Italy

32 Istituto Nazionale di Fisica Nucleare, Sezione di Pisa, I-56127 Pisa, Italy

${ }^{33}$ Laboratoire AIM, CEA-IRFU/CNRS/Université Paris Diderot, Service d'Astrophysique, CEA Saclay, 91191 Gif sur Yvette, France

${ }^{34}$ Istituto Nazionale di Fisica Nucleare, Sezione di Trieste, I-34127 Trieste, Italy

${ }^{35}$ Dipartimento di Fisica, Università di Trieste, I-34127 Trieste, Italy

${ }^{36}$ Istituto Nazionale di Fisica Nucleare, Sezione di Padova, I-35131 Padova, Italy

${ }^{37}$ Dipartimento di Fisica "G. Galilei," Università di Padova, I-35131 Padova, Italy

${ }^{38}$ Dipartimento di Fisica “M. Merlin" dell’Università e del Politecnico di Bari, I-70126 Bari, Italy

${ }^{39}$ Istituto Nazionale di Fisica Nucleare, Sezione di Bari, 70126 Bari, Italy

${ }^{40}$ Laboratoire Leprince-Ringuet, École polytechnique, CNRS/IN2P3, Palaiseau, France; fortin@1lr.in2p3.fr

${ }^{41}$ Institut de Ciencies de l'Espai (IEEC-CSIC), Campus UAB, 08193 Barcelona, Spain

42 INAF-Istituto di Astrofisica Spaziale e Fisica Cosmica, I-20133 Milano, Italy

${ }^{43}$ Agenzia Spaziale Italiana (ASI) Science Data Center, I-00044 Frascati (Roma), Italy

${ }^{44}$ NASA Goddard Space Flight Center, Greenbelt, MD 20771, USA

${ }^{45}$ Center for Research and Exploration in Space Science and Technology (CRESST) and NASA Goddard Space Flight Center, Greenbelt, MD 20771, USA

${ }^{46}$ Department of Physics and Center for Space Sciences and Technology, University of Maryland Baltimore County, Baltimore, MD 21250, USA

${ }^{47}$ Space Science Division, Naval Research Laboratory, Washington, DC 20375, USA

${ }^{48}$ George Mason University, Fairfax, VA 22030, USA

${ }^{49}$ National Research Council Research Associate, National Academy of Sciences, Washington, DC 20001, USA

${ }^{50}$ Laboratoire de Physique Théorique et Astroparticules, Université Montpellier 2, CNRS/IN2P3, Montpellier, France

${ }^{51}$ Department of Physics, Stockholm University, AlbaNova, SE-106 91 Stockholm, Sweden

52 The Oskar Klein Centre for Cosmoparticle Physics, AlbaNova, SE-106 91 Stockholm, Sweden

${ }^{53}$ CNRS/IN2P3, Centre d'Études Nucléaires Bordeaux Gradignan, UMR 5797, Gradignan, 33175, France

${ }^{54}$ Centre d'Études Nucléaires Bordeaux Gradignan, Université de Bordeaux, UMR 5797, Gradignan, 33175, France

${ }^{55}$ Dipartimento di Fisica, Università di Udine and Istituto Nazionale di Fisica Nucleare, Sezione di Trieste, Gruppo Collegato di Udine, I-33100 Udine, Italy

${ }^{56}$ Osservatorio Astronomico di Trieste, Istituto Nazionale di Astrofisica, I-34143 Trieste, Italy

${ }^{57}$ Department of Physical Sciences, Hiroshima University, Higashi-Hiroshima, Hiroshima 739-8526, Japan

${ }^{58}$ INAF Istituto di Radioastronomia, 40129 Bologna, Italy

${ }^{59}$ Center for Space Plasma and Aeronomic Research (CSPAR), University of Alabama in Huntsville, Huntsville, AL 35899, USA

${ }^{60}$ Department of Physics, Center for Cosmology and Astro-Particle Physics, The Ohio State University, Columbus, OH 43210, USA

${ }^{61}$ Research Institute for Science and Engineering, Waseda University, 3-4-1, Okubo, Shinjuku, Tokyo 169-8555, Japan

${ }^{62}$ Centre d'Étude Spatiale des Rayonnements, CNRS/UPS, BP 44346, F-30128 Toulouse Cedex 4, France

${ }^{63}$ Department of Physics and Department of Astronomy, University of Maryland, College Park, MD 20742, USA

${ }^{64}$ Istituto Nazionale di Fisica Nucleare, Sezione di Roma "Tor Vergata," I-00133 Roma, Italy

${ }^{65}$ Department of Physics and Astronomy, University of Denver, Denver, CO 80208, USA

${ }^{66}$ Institute of Space and Astronautical Science, JAXA, 3-1-1 Yoshinodai, Sagamihara, Kanagawa 229-8510, Japan

${ }^{67}$ Hiroshima Astrophysical Science Center, Hiroshima University, Higashi-Hiroshima, Hiroshima 739-8526, Japan ${ }^{68}$ Max-Planck Institut für extraterrestrische Physik, 85748 Garching, Germany

${ }^{69}$ Institut für Astro- und Teilchenphysik and Institut für Theoretische Physik, Leopold-Franzens-Universität Innsbruck, A-6020 Innsbruck, Austria

70 Department of Physics, University of Washington, Seattle, WA 98195-1560, USA

${ }^{71}$ Santa Cruz Institute for Particle Physics, Department of Physics and Department of Astronomy and Astrophysics, University of California at Santa Cruz, Santa Cruz, CA 95064, USA

${ }^{72}$ Space Sciences Division, NASA Ames Research Center, Moffett Field, CA 94035-1000, USA

${ }^{73}$ NYCB Real-Time Computing Inc., Lattingtown, NY 11560-1025, USA

74 Department of Chemistry and Physics, Purdue University Calumet, Hammond, IN 46323-2094, USA

${ }^{75}$ Institució Catalana de Recerca i Estudis Avançats (ICREA), Barcelona, Spain

${ }^{76}$ Consorzio Interuniversitario per la Fisica Spaziale (CIFS), I-10133 Torino, Italy

77 INTEGRAL Science Data Centre, CH-1290 Versoix, Switzerland

${ }^{78}$ Dipartimento di Fisica, Università di Roma "Tor Vergata," I-00133 Roma, Italy

79 Department of Physics, Royal Institute of Technology (KTH), AlbaNova, SE-106 91 Stockholm, Sweden

${ }^{80}$ School of Pure and Applied Natural Sciences, University of Kalmar, SE-391 82 Kalmar, Sweden Received 2010 March 12; accepted 2010 April 21; published 2010 April 30

\section{ABSTRACT}

The high-frequency-peaked BL Lacertae object RGB J0710+591 was observed in the very high-energy (VHE; $E>100 \mathrm{GeV}$ ) wave band by the VERITAS array of atmospheric Cherenkov telescopes. The observations, taken between 2008 December and 2009 March and totaling $22.1 \mathrm{hr}$, yield the discovery of VHE gamma rays from the source. RGB J0710+591 is detected at a statistical significance of 5.5 standard deviations $(5.5 \sigma)$ above the background, corresponding to an integral flux of $(3.9 \pm 0.8) \times 10^{-12} \mathrm{~cm}^{-2} \mathrm{~s}^{-1}$ (3\% of the Crab Nebula's flux) above $300 \mathrm{GeV}$. The observed spectrum can be fit by a power law from 0.31 to $4.6 \mathrm{TeV}$ with a photon spectral index of $2.69 \pm 0.26_{\text {stat }} \pm 0.20_{\text {sys. }}$. These data are complemented by contemporaneous multiwavelength data from the Fermi Large Area Telescope, the Swift X-ray Telescope, the Swift Ultra-Violet and Optical Telescope, and the Michigan-Dartmouth-MIT observatory. Modeling the broadband spectral energy distribution (SED) with an equilibrium synchrotron self-Compton model yields a good statistical fit to the data. The addition of an external-Compton component to the model does not improve the fit nor brings the system closer to equipartition. The combined Fermi and VERITAS data constrain the properties of the high-energy emission component of the source over 4 orders of magnitude and give measurements of the rising and falling sections of the SED. Key words: BL Lacertae objects: individual (RGB J0710+591, VER J0710+591) - gamma rays: galaxies

Online-only material: color figure 


\section{INTRODUCTION}

RGB J0710+591 was originally discovered by HEAO A-1 (Wood et al. 1984); it is a BL Lac object located at a redshift of $z=0.125$ (Giommi et al. 1991). Hubble Space Telescope observations of this blazar show a fully resolved diskless galaxy that can be modeled using an elliptical profile with a nuclear point source (Scarpa et al. 2000). In addition, RGB J0710+591 is found to have an optical "compact companion" located at a distance of 1".4 (3.5 kpc) from the core of the active galactic nucleus. The nature of this companion is unknown, but it could be a foreground star or an unresolved faint galaxy. It is, however, too luminous for a globular cluster (Falomo et al. 2000) and is not seen in radio (Giroletti et al. 2004).

Blazars are characterized by spectral energy distributions (SEDs) consisting of two peaks, the low-energy peak arising from synchrotron emission and the high-energy (HE) peak from either leptonic or hadronic interactions (for a review of the emission mechanisms in blazars, see Böttcher 2007). Depending on the location of the low-energy peak, BL Lac objects are classified as being high-frequency-peaked (HBL; $v_{\text {peak }} \sim 10^{16}-10^{18} \mathrm{~Hz}$ ), intermediate-frequency-peaked (IBL; $\left.v_{\text {peak }} \sim 10^{15}-10^{16} \mathrm{~Hz}\right)$, or low-frequency-peaked (LBL; $v_{\text {peak }} \sim$ $10^{13}-10^{15} \mathrm{~Hz}$ ). The location of the low-energy SED peak of RGB J0710+591 (identified as EXO 0706.1+5913 in the following reference) is higher than $\sim 10^{18} \mathrm{~Hz}$ (Nieppola et al. 2006), clearly identifying it as an HBL.

RGB J0710+591 is a well-known HBL featured in many catalogs, but it was not detected in the $\mathrm{HE}(E>100 \mathrm{MeV})$ band by the EGRET instrument on the Compton Gamma-ray Observatory. In the very high-energy (VHE; $E>100 \mathrm{GeV}$ ) band, the Whipple $10 \mathrm{~m}$ imaging atmospheric Cherenkov telescope (IACT) reported several flux upper limits for observations taken in different epochs: $(0.91,1.69,3.58$, and 4.29) $\times 10^{-12} \mathrm{~cm}^{-2} \mathrm{~s}^{-1}(99.9 \%$ confidence level) above $(350,350$, 500 , and $400 \mathrm{GeV})$, respectively, corresponding to $(8.7 \%$, $16.1 \%, 52.4 \%$, and $45.9 \%$ ) of the Crab Nebula's flux (Horan et al. 2004). The HEGRA IACT system reported a flux upper limit above $1.08 \mathrm{TeV}$ of $0.91 \times 10^{-12} \mathrm{~cm}^{-2} \mathrm{~s}^{-1}$ (99\% confidence level) corresponding to $6 \%$ of the Crab Nebula's flux (Aharonian et al. 2004).

There are several compelling reasons to study RGB J0710+ 591 in the $\mathrm{MeV}-\mathrm{TeV}$ energy range. Due to the hardness of its $\mathrm{X}$-ray spectrum, the HE peak is expected to be located in an ideal location for HE and VHE observations. There are both leptonic (Ghisellini \& Madau 1996; Böttcher \& Chiang 2002) and hadronic models (Aharonian 2000; Mücke et al. 2003) that explain the photon-production mechanism of the HE peak. The continuous measurement of the SED from $\mathrm{MeV}$ to $\mathrm{TeV}$ energies is vital to constrain certain aspects of the emission models and to perhaps exclude some of them entirely. For example, some leptonic models appear harder at HEs than hadronic models. At VHE energies, photons interact via pair-production with the infrared component of the extragalactic background light

\footnotetext{
${ }^{81}$ Current address: DESY, Platanenallee 6, 15738 Zeuthen, Germany.

${ }^{82}$ Current address: Institut für Physik und Astronomie, Universität Potsdam, 14476 Potsdam-Golm, Germany; DESY, Platanenallee 6, 15738 Zeuthen, Germany.

${ }^{83}$ Current address: Los Alamos National Laboratory, MS H803, Los Alamos, NM 87545, USA.

${ }^{84}$ Royal Swedish Academy of Sciences Research Fellow, funded by a grant from the K. A. Wallenberg Foundation.

${ }^{85}$ Partially supported by the International Doctorate on Astroparticle Physics (IDAPP) program.
}

(EBL; Gould \& Schréder 1967). This causes a decrease in the observed flux and a softening of the observed spectrum. Thus, extragalactic objects at moderate redshifts with reasonably hard VHE spectra are ideal candidates to indirectly study the history of the EBL and its SED, particularly in the optical to far infrared (see, e.g., Aharonian et al. 2007).

VHE $\gamma$-ray emission from RGB J0710+591 was discovered by VERITAS during the 2008-2009 observing season (Ong et al. 2009). Following this, the VERITAS collaboration initiated a multiwavelength (MWL) observation campaign that included the participation of the Fermi Large Area Telescope (LAT) in HE $\gamma$-rays, the Swift X-ray Telescope (XRT) in X-rays, and the Swift Ultra-Violet and Optical Telescope (UVOT) and the Michigan-Dartmouth-MIT (MDM) observatory in optical. The resulting MWL SED spans 13 decades in energy.

\section{VERITAS OBSERVATIONS}

VERITAS is an array of four $12 \mathrm{~m}$ IACTs located in southern Arizona at the F. L. Whipple Observatory (Weekes et al. 2002; Holder et al. 2008). VERITAS has an energy resolution of $\sim 15 \%$ between $100 \mathrm{GeV}$ and $30 \mathrm{TeV}$, an angular resolution of $\sim 0.1$ (68\% containment) per event, and a field of view (FoV) of 3.5. VERITAS can detect a source with a flux of $1 \%$ of the Crab in $<50 \mathrm{hr}$, while a 5\% Crab Nebula flux source is detected in $\sim 2.5 \mathrm{hr}$. These specifications are valid for the data taken during this campaign; the VERITAS array was subsequently upgraded, resulting in a significant improvement in sensitivity (Perkins et al. 2009).

RGB J0710+591 was observed with VERITAS from 2008 December to 2009 March during astronomical darkness and partial moonlight for a total time of $34.9 \mathrm{hr}$. Approximately $30 \%$ of these observations are rejected due to poor weather or hardware-related problems with the majority rejected due to weather, resulting in $22.1 \mathrm{hr}$ of exposure (livetime corrected). The average zenith angle of the observations is $30^{\circ}$. The VERITAS data are calibrated and cleaned as described in Daniel et al. (2008). After calibration, several noise-reducing selection criteria (cuts) are made, including rejecting those events where only the two closest-spaced telescopes participated in the trigger. The VERITAS standard analysis consists of stereo parameterization using a moment analysis (Hillas 1985) and following this, scaled parameters are used for event selection (Aharonian et al. 1997; Krawczynski et al. 2006). This selection is based on a set of cuts that are a priori optimized using data taken on the Crab Nebula where the $\gamma$-ray excess has been scaled by 0.01 to account for weaker sources like RGB J0710+591. VERITAS observed RGB J0710+591 in "wobble" mode, where the center of the FoV is offset by 0.5 from the blazar, to allow for simultaneous source and background measurements (Berge et al. 2007). The reflected region model (Aharonian et al. 2001) is used to calculate the background events from several off-source regions. A total of 576 on-source events are observed from the direction of RGB J0710+591 and 3890 are seen in the background regions. Depending on the pointing direction either eight or nine background regions are used resulting in a final $\alpha$ of 0.115 (here $\alpha$ is the ratio of the areas of the on- and off-source regions). This results in an excess of 129 events. This excess has a statistical significance of 5.5 standard deviations $(\sigma)$ and corresponds to an integral flux of $(3.9 \pm 0.8) \times 10^{-12} \mathrm{~cm}^{-2} \mathrm{~s}^{-1}$ (3\% of the Crab Nebula's flux) above $300 \mathrm{GeV}$. There is no evidence for variability during these observations (a fit of the light curve to a constant flux yields a reduced chi-squared value of 0.21 ). The excess is 


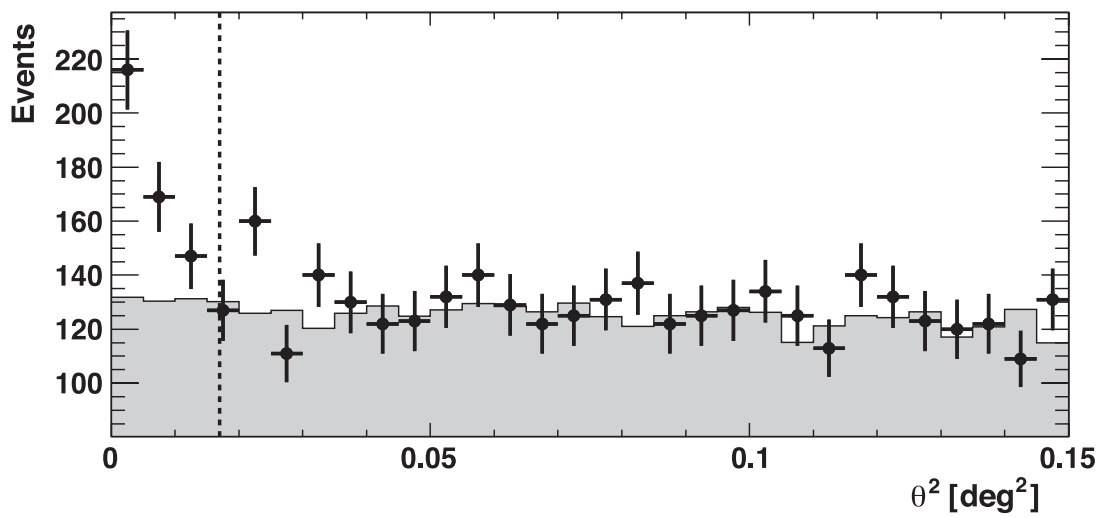

Figure 1. Distribution of $\theta^{2}$ for on-source VHE events (point, shaded region) from observations of RGB J0710+591. $\theta$ is the distance from the source location to the event location. The dashed line represents the location of the cut on $\theta^{2}$ applied to the data. The $\theta^{2}$ cut retains events which lie to the left of the line.

Table 1

The VERITAS Spectral Bins

\begin{tabular}{lcccrr}
\hline \hline $\begin{array}{c}E_{\text {low }} \\
(\mathrm{TeV})\end{array}$ & $\begin{array}{c}E_{\text {high }} \\
(\mathrm{TeV})\end{array}$ & $\begin{array}{c}\text { Flux } \\
\left(\mathrm{cm}^{-2} \mathrm{TeV}^{-1} \mathrm{~s}^{-1}\right)\end{array}$ & $\begin{array}{c}\text { Flux Error } \\
\left(\mathrm{cm}^{-2} \mathrm{TeV}^{-1} \mathrm{~s}^{-1}\right)\end{array}$ & Excess & $\begin{array}{r}\text { Sig. } \\
(\sigma)\end{array}$ \\
\hline 0.31 & 0.53 & $8.9 \times 10^{-12}$ & $3.6 \times 10^{-12}$ & 29.9 & 2.7 \\
0.53 & 0.92 & $2.4 \times 10^{-12}$ & $7.7 \times 10^{-13}$ & 32.9 & 3.5 \\
0.92 & 1.6 & $7.3 \times 10^{-13}$ & $2.2 \times 10^{-13}$ & 24.3 & 3.9 \\
1.6 & 2.7 & $8.6 \times 10^{-14}$ & $5.6 \times 10^{-14}$ & 6.07 & 1.7 \\
2.7 & 4.6 & $3.1 \times 10^{-14}$ & $2.0 \times 10^{-14}$ & 4.25 & 2.0 \\
4.6 & 7.9 & $7.4 \times 10^{-15}$ & $99 \%$ upper limit & -0.26 & -0.23 \\
\hline
\end{tabular}

Notes. " $E_{\text {low" }}$ " and " $E_{\text {high" }}$ are the low- and high-energy bounds; "Flux" is the differential flux; "Excess" is the number of excess events; "Sig." is the significance of the bin.

point like and is located at $07^{\mathrm{h}} 10^{\mathrm{m}} 26^{\mathrm{s}} 4 \pm 2.4_{\text {stat }}^{\mathrm{s}}, 59^{\circ} 09^{\prime} 00^{\prime \prime} \pm$ $36_{\text {stat }}^{\prime \prime}$ (J2000, see Figure 2; the systematic uncertainty in the pointing is $90^{\prime \prime}$ ) which is $49^{\prime \prime}$ away from the location of RGB J0710+591 $\left(07^{\mathrm{h}} 10^{\mathrm{m}} 30^{\mathrm{s}} .07,59^{\circ} 08^{\prime} 20^{\prime} \cdot 5\right)$ measured by the Very Large Array (VLA) at $5 \mathrm{GHz}$ (Laurent-Muehleisen et al. 1999). The VERITAS source name is VER J0710+591. The $\theta^{2}$ distributions for the on-source and background regions are shown in Figure 1 (here, $\theta$ is the distance between the source location and the location of an event). The photon energy spectrum can be fit by a simple power law $(d N / d E=$ $\left.I\left(E / E_{0}\right)^{-\Gamma}, E_{0}=1 \mathrm{TeV}\right)$ with photon index $\Gamma_{\mathrm{VHE}}=2.69 \pm$ $0.26_{\text {stat }} \pm 0.20_{\text {sys }}$ (see Table 1 for the spectral points). The fit yields a $\chi^{2}$ per degree of freedom of $\chi^{2} /$ dof $=1.3 / 3$.

\section{FERMI OBSERVATIONS}

The Fermi LAT is a pair-conversion gamma-ray detector sensitive to photons in the energy range from below $20 \mathrm{MeV}$ to more than $300 \mathrm{GeV}$ (Atwood et al. 2009). The LAT data for this analysis were taken between 2008 August 4 and 2009 August 4 . The data are analyzed using the standard likelihood tools distributed with the Science Tools v9r15p3 package available from the Fermi Science Support Center. ${ }^{86}$ Only events having the highest probability of being photons, those in the "diffuse" class, are used. To limit contamination by Earth albedo gamma rays which are produced by cosmic rays interacting with the upper atmosphere, only events with zenith angles $<105^{\circ}$ are selected. Events with energy between $100 \mathrm{MeV}$ and $300 \mathrm{GeV}$ and within a circular region of $10^{\circ}$ radius centered on the VLA

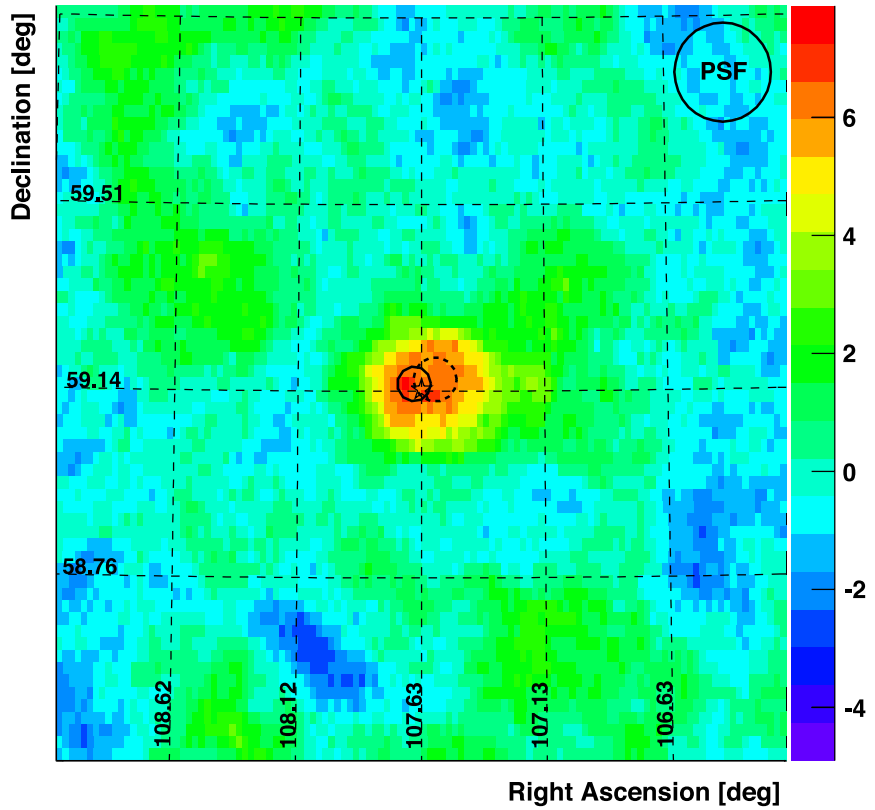

Figure 2. Smoothed VHE significance map of RGB J0710+591 from the VERITAS observations. The VLA location of the blazar is shown as a star. The solid circle is centered at the best fit location of the excess emission in the VERITAS data; the radius of the circle is the sum of the statistical and systematic uncertainties. The dashed circle is the best fit plus a 95\% containment radius of 2.7 to the Fermi LAT data. Both locations are consistent with the VLA position of RGB J0710+591. The VERITAS point-spread function (PSF) is shown in the top right corner.

(A color version of this figure is available in the online journal.)

coordinates of RGB J0710+591 are analyzed with an unbinned maximum likelihood method (Cash 1979; Mattox et al. 1996). The background emission is modeled using a Galactic diffuse emission model and an isotropic component. ${ }^{87}$ The fluxes are determined using the post-launch instrument response functions P6_V3_DIFFUSE.

A point source is detected with a significance of more than eight standard deviations. The best-fit position $\left(07^{\mathrm{h}} 10^{\mathrm{m}} 37^{\mathrm{s}}\right.$, $59^{\circ} 09^{\prime} 32^{\prime \prime}$ ) has a $95 \%$ containment radius of 2.7 and is statistically consistent with the VLA coordinates of RGB J0710+591 (see Figure 2). The highest energy photon associated with the source has an energy of $75 \mathrm{GeV}$. The time-averaged Fermi spectrum is fit by a power law for which $I=1.43 \pm 0.35_{\text {stat }} \pm$ 
$0.10_{\text {sys }} \times 10^{-14} \mathrm{~cm}^{-2} \mathrm{~s}^{-1} \mathrm{MeV}^{-1}, \Gamma_{\mathrm{HE}}=1.46 \pm 0.17_{\text {stat }} \pm$ $0.05_{\text {sys }}$, and $E_{0}=8.8 \mathrm{GeV}$ is the energy at which the correlation between the fit values of $\mathrm{I}$ and $\Gamma_{\mathrm{HE}}$ is minimized. The spectral index reported in the 1FGL catalog (Abdo et al. 2010) for this source is $1.28 \pm 0.21_{\text {stat }}$. The discrepancy between that result and the result reported here can be explained by the different energy range considered in the likelihood model of the source: $100 \mathrm{MeV}-100 \mathrm{GeV}$ for the catalog analysis, $100 \mathrm{MeV}-300 \mathrm{GeV}$ for the present analysis. The lack of photons above $75 \mathrm{GeV}$ carries information that is important for the fit results. No evidence for variability is detected.

\section{SWIFT OBSERVATIONS}

A total of seven Swift (Gehrels et al. 2004) XRT and UVOT observations of RGB J0710+591 were performed between 2009 February 20 and 2009 March 2 (observation identifications 00031356001 through 00031356007$)$. The total XRT observation time is $15.2 \mathrm{ks}$ while the total UVOT observation time is 14.8 ks. The Swift XRT data are analyzed with HEAsoft 6.5 using the most recent calibration files. The first three XRT observations in photon-counting mode have source count rates of $\sim 0.7-1.5$ counts $\mathrm{s}^{-1}$, resulting in significant photon pile-up in the core of the point-spread function (PSF), which occurs for rates $>0.5$ counts $\mathrm{s}^{-1}$. The photon pile-up is avoided by using an annular region with inner radius ranging from 2 to 7 pixels and an outer radius of 30 pixels (47".2) to extract the source counts. Background counts are extracted from a 40 pixel radius circle in a source-free region. For the last four XRT observations in windowed-timing mode, source counts are extracted from a rectangular region of 40 by 20 pixels high, with background counts extracted from a nearby source-free rectangular region of equivalent size. Evidence for marginal flux variability between the Swift XRT observations is indicated by a probability of 0.004 for a fit of a constant flux. The time-averaged XRT data can be fit with an absorbed power-law model where the hydrogen column density $N_{\mathrm{H}}$ is fixed at $4.4 \times 10^{20} \mathrm{~cm}^{-2}$ (Kalberla et al. 2005), resulting in a combined spectrum with a photon index of $1.86 \pm 0.01$, with a $\chi^{2} / \mathrm{dof}=371 / 303$. This hard spectrum is consistent with a synchrotron peak above $10 \mathrm{keV}$, as can be inferred from the radio to X-ray SED generated from archival data, including an EXOSAT detection (Giommi et al. 1995).

Swift UVOT (Roming et al. 2005) data are presented in the six bands of $v, b, u, u v m 1, u v m 2$, and $u v w 2$. The UVOTSOURCE tool is used to extract counts, correct for coincidence losses, apply background subtraction, and calculate the source flux (Poole et al. 2008). The standard $5^{\prime \prime}$ radius source aperture is used, with a $20^{\prime \prime}$ background region. The source fluxes are extinction corrected using the dust maps of Schlegel et al. (1998). Since the stacked UVOT images are not deep enough to sample the host galaxy profile, no attempt is made to correct for the contribution of the host galaxy and this can be seen as an increase in flux in the optical portion of the SED (Figure 3) starting with the $u$ band.

\section{MDM OBSERVATIONS}

The observations of RGB J0710+591 taken from 2009 February 19 through 24 with the MDM Observatory $1.3 \mathrm{~m}$ telescope ${ }^{88}$ in $R$ and $B$ bands are bias-subtracted and flatfielded using the Image Reduction and Analysis Facility (IRAF;

\footnotetext{
88 http://www.astro.lsa.umich.edu/obs/mdm/
}

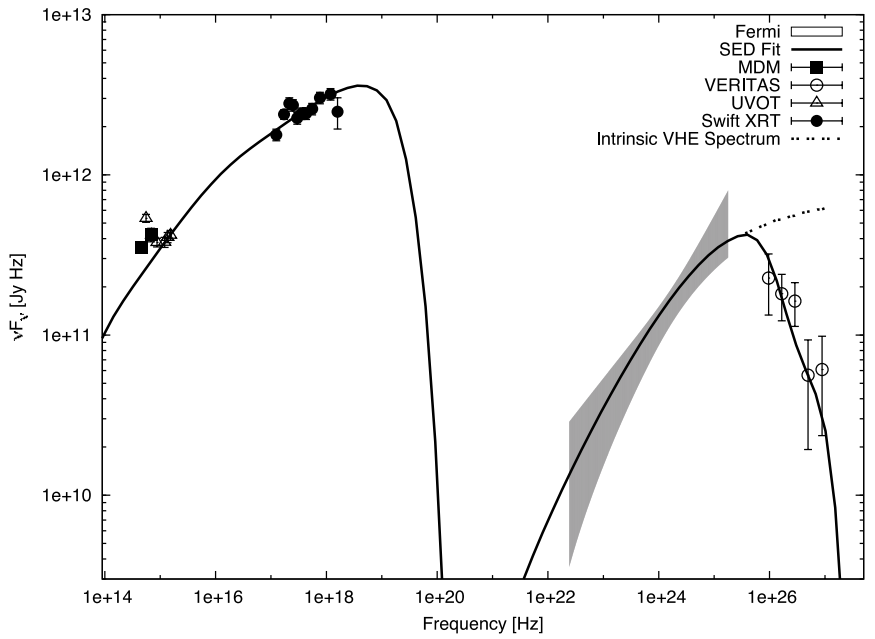

Figure 3. Broadband spectral energy distribution (SED) of RGB J0710+591. The closed squares are $R$ - and $B$-band data from the MDM $1.3 \mathrm{~m}$ telescope; the open triangles are from the Swift UVOT; closed circles are from the Swift XRT; the shaded area is the Fermi LAT measurement; the open circles are the measured VERITAS data. The broadband spectral model is the equilibrium SSC version from Böttcher \& Chiang (2002) with a cooling break energy occurring around $1.3 \times 10^{16} \mathrm{~Hz}$. Note that the optical measurements (MDM and Swift $u$, $b$ and $v$ bands) are affected by emission from the host galaxy and are thus not used in the SED fit. The SED model curve includes EBL absorption using the model of Franceschini et al. (2008), and the dashed line is the de-absorbed VHE spectrum.

Tody 1993, 1986). Instrumental magnitudes are extracted using the IRAF DAOPHOT package and calibrated using four comparison stars in the field, with comparison star magnitudes from the Naval Observatory Merged Astrometric Dataset (NOMAD) catalog (Zacharias et al. 2004), and extinction coefficients $A_{B}=0.166, A_{R}=0.106$ from the NASA/IPAC Extragalactic Database (NED). ${ }^{89}$

The host galaxy of RGB J0710+591 makes a significant contribution to the optical fluxes measured by MDM. The host galaxy subtractions for this BL Lac object were calculated previously in Hyvönen et al. (2007) and these subtractions are used to correct for the host galaxy contribution to the flux measurements. The resulting extinction- and host-galaxycorrected optical flux in the $R$ band $\left(4.68 \times 10^{14} \mathrm{~Hz}\right)$ is $(3.51 \pm 0.07) \times 10^{11} \mathrm{Jy} \mathrm{Hz}$ and in the $B$ band $\left(6.85 \times 10^{14} \mathrm{~Hz}\right)$ is $(4.23 \pm 0.41) \times 10^{11} \mathrm{JyHz}$. Even with the host galaxy corrections, the MDM data are still higher than the UV measurements made by the Swift UVOT which are dominated by the nucleus. Thus, the MDM and Swift optical data in the $u, b$, and $v$ bands still have contributions due to the host galaxy and should be considered upper limits. No substantial optical variability is seen over the course of the 5 day observation period.

\section{MODELING AND DISCUSSION}

The observed broadband SED of RGB J0710+591 is shown in Figure 3. The data, excluding the MDM and Swift $u, b$, and $v$ bands which are affected by contributions from the host, are fit with a purely leptonic synchrotron self-Compton (SSC) model. Specifically, an equilibrium version of the model of Böttcher \& Chiang (2002), as described in Acciari et al. (2009), is used. The best-fit parameters are listed in Table 2. The VHE data in the plot are the measured values and the model includes the effects of intergalactic $\gamma-\gamma$ absorption using the EBL models of

\footnotetext{
89 http://nedwww.ipac.caltech.edu/
} 
Table 2

The Equilibrium Model Parameters for the Purely Leptonic SSC Model That Was Used to Fit the SED of RGB J0170+591

\begin{tabular}{lc}
\hline \multicolumn{1}{c}{ Parameter } & Value \\
\hline$\gamma_{\min }$ & $6 \times 10^{4}$ \\
$\gamma_{\max }$ & $2 \times 10^{6}$ \\
$e^{- \text {injection spectral index }}$ & 1.5 \\
Escape time parameter & $\eta_{\mathrm{esc}}=100^{\mathrm{a}}$ \\
Magnetic field at $z_{0}$ & $0.036 \mathrm{G}$ \\
Bulk Lorentz factor & $\Gamma_{\text {bulk }}=30$ \\
Blob radius & $2 \times 10^{16} \mathrm{~cm}$ \\
$\theta_{\text {obs }}$ & $1.91^{\mathrm{b}}$ \\
Redshift & $z=0.125$ \\
$L_{e}($ jet $)$ & $4.49 \times 10^{43} \mathrm{erg} \mathrm{s}^{-1}$ \\
$L_{B}($ jet $)$ & $1.75 \times 10^{42} \mathrm{erg} \mathrm{s}^{-1}$ \\
$L_{B} / L_{e}$ & $3.90 \times 10^{-2}$ \\
\hline
\end{tabular}

Notes. Details about the model can be found in the text.

${ }^{\mathrm{a}} t_{\mathrm{esc}}=\eta_{\mathrm{esc}} * R / c$.

$\mathrm{b}$ Equivalent to the superluminal angle.

Franceschini et al. (2008) and the reported redshift of $z=0.125$. De-absorbing the VHE data using the same models results in a de-absorbed spectral index of $\Gamma_{\mathrm{VHE}} \sim 1.85$.

Many of the model parameters are not constrained by the observations. In particular, the choice of the bulk Lorentz factor $\left(\Gamma_{\text {bulk }}\right)$ and observing angle $\left(\theta_{\text {obs }}\right)$ are somewhat arbitrary and similarly good fits can be obtained using other values. Evidence from portions of the radio structure is supportive of a small observing angle which corroborates the small value obtained here. However, the bulk Lorentz factor derived from these same data is lower (Giroletti et al. 2006). Regardless, the synchrotron portion of the SED always turns over far below the X-ray regime. Therefore, in order to obtain the observed hard X-ray spectrum, a very hard injection index $(q=1.5)$ has to be assumed. This poses challenges to standard (first-order) Fermi acceleration models (Stecker et al. 2007), and it might indicate second-order Fermi acceleration or other modes of acceleration (e.g., shear at boundary layers). The fit requires a rather low magnetic field of $B=0.036 \mathrm{G}$, far below equipartition. This cannot be remedied even with the choice of a higher Doppler factor.

Additionally, an external-Compton (EC) component was added to the model to see if the resulting fit would improve, or if the system would be closer to equipartition. However, in order to produce an EC peak at sufficiently high frequencies, a very low magnetic field is needed. Thus, the inclusion of an EC component does not lead to an overall improvement of the fit statistically, or with respect to equipartition.

With a photon index $\Gamma_{\mathrm{VHE}}=2.69$, RGB J0710+591 is one of the hardest VHE blazars detected to date. Furthermore, out of the 19 blazars detected by both VHE instruments and Fermi, RGB J0710+591 has the hardest HE photon index (Abdo et al. 2009) and Nieppola et al. (2006) found that RGB J0710+591 has one of the highest low-energy SED peaks out of a sample of over $300 \mathrm{BL}$ Lac objects and place it in a list of 22 ultra-highfrequency-peaked candidates. Nevertheless, the observed SED is well fit by a purely leptonic SSC model without the need to include an external component.

Observations of the similarly hard $\left(\Gamma_{\mathrm{VHE}}=2.50\right)$ and distant $(z=0.1396)$ blazar 1ES 0229+200 (Aharonian et al. 2007) indicated that the EBL density in the mid-IR band was close to the lower limits measured by Spitzer. These constraints on the EBL are based on the assumption that the intrinsic spectrum of blazars must have a spectral index of 1.5 or larger.
Thus, if blazars like 1ES 0229+200 and RGB J0710+591 are unusual objects, this assumption might not be valid because their intrinsic spectral indices could be smaller than 1.5. However, the modeling of RGB J0710+591 and other similar blazars indicates that hard-spectrum VHE blazars might not be exotic (i.e., having an intrinsic spectral index less than 1.5) and are part of the general blazar population. Therefore, using VHE observations of hard spectrum blazars to constrain EBL models as done in Aharonian et al. (2007) continues to be a valid undertaking.

The VERITAS Collaboration acknowledges support from the U.S. Department of Energy, the U.S. National Science Foundation and the Smithsonian Institution, by NSERC in Canada, by Science Foundation Ireland, and by STFC in the UK. This research has made use of the NASA/IPAC Extragalactic Database (NED) which is operated by the Jet Propulsion Laboratory, California Institute of Technology, under contract with the National Aeronautics and Space Administration.

The Fermi LAT Collaboration acknowledges generous ongoing support from a number of agencies and institutes that have supported both the development and the operation of the LAT as well as scientific data analysis. These include the National Aeronautics and Space Administration and the Department of Energy in the United States, the Commissariat à l'Energie Atomique and the Centre National de la Recherche Scientifique/ Institut National de Physique Nucléaire et de Physique des Particules in France, the Agenzia Spaziale Italiana and the Istituto Nazionale di Fisica Nucleare in Italy, the Ministry of Education, Culture, Sports, Science, and Technology (MEXT), High Energy Accelerator Research Organization (KEK) and Japan Aerospace Exploration Agency (JAXA) in Japan, and the K. A. Wallenberg Foundation, the Swedish Research Council, and the Swedish National Space Board in Sweden.

Additional support for science analysis during the operations phase is gratefully acknowledged from the Istituto Nazionale di Astrofisica in Italy and the Centre National d'Études Spatiales in France.

\section{REFERENCES}

Abdo, A. A., et al. 2009, ApJ, 707, 1310

Abdo, A. A., et al. 2010, ApJS, in press (arXiv:1002.2280)

Acciari, V. A., et al. 2009, ApJ, 707, 612

Aharonian, F. A. 2000, New Astron., 5, 377

Aharonian, F. A., Hofmann, W., Konopelko, A. K., \& Völk, H. J. 1997, Astropart. Phys., 6, 343

Aharonian, F., et al. 2001, A\&A, 370, 112

Aharonian, F., et al. 2004, A\&A, 421, 529

Aharonian, F., et al. 2007, A\&A, 475, L9

Atwood, W. B., et al. 2009, ApJ, 697, 1071

Berge, D., Funk, S., \& Hinton, J. 2007, A\&A, 466, 1219

Böttcher, M. 2007, Ap\&SS, 309, 95

Böttcher, M., \& Chiang, J. 2002, ApJ, 581, 127

Cash, W. 1979, ApJ, 228, 939

Daniel, M. K., et al. 2008, Proc. ICRC, 3, 1325

Falomo, R., Scarpa, R., Treves, A., \& Urry, C. M. 2000, ApJ, 542, 731 Franceschini, A., Rodighiero, G., \& Vaccari, M. 2008, A\&A, 487, 837

Gehrels, N., et al. 2004, ApJ, 611, 1005

Ghisellini, G., \& Madau, P. 1996, MNRAS, 280, 67

Giommi, P., Ansari, S. G., \& Micol, A. 1995, A\&AS, 109, 267

Giommi, P., et al. 1991, ApJ, 378, 77

Giroletti, M., Giovannini, G., Taylor, G. B., \& Falomo, R. 2004, ApJ, 613, 752

Giroletti, M., Giovannini, G., Taylor, G. B., \& Falomo, R. 2006, ApJ, 646, 801 Gould, R. J., \& Schréder, G. P. 1967, Phys. Rev., 155, 1408

Hillas, A. M. 1985, Proc. ICRC, 3, 445

Holder, J., et al. 2008, in AIP Conf. Ser. 1085, High Energy Gamma-ray Astronomy, ed. F. A. Aharonian, W. Hofmann, \& F. Rieger (Melville, NY: AIP), 657 
Horan, D., et al. 2004, ApJ, 603, 51

Hyvönen, T., Kotilainen, J. K., Falomo, R., Örndahl, E., \& Pursimo, T. 2007, A\&A, 476, 723

Kalberla, P. M. W., Burton, W. B., Hartmann, D., Arnal, E. M., Bajaja, E., Morras, R., \& Pöppel, W. G. L. 2005, A\&A, 440, 775

Krawczynski, H., Carter-Lewis, D. A., Duke, C., Holder, J., Maier, G., Le Bohec, S., \& Sembroski, G. 2006, Astropart. Phys., 25, 380

Laurent-Muehleisen, S. A., Kollgaard, R. I., Feigelson, E. D., Brinkmann, W., \& Siebert, J. 1999, ApJ, 525, 127

Mattox, J. R., et al. 1996, ApJ, 461, 396

Mücke, A., Protheroe, R. J., Engel, R., Rachen, J. P., \& Stanev, T. 2003, Astropart. Phys., 18, 593

Nieppola, E., Tornikoski, M., \& Valtaoja, E. 2006, A\&A, 445, 441

Ong, R. A., et al. 2009, ATel, 1941

Perkins, J. S., et al. 2009, in Proc. 2009 Fermi Symposium, ed. D. J. Thompson (Palo Alto, CA: Stanford Univ.), eConf, C091122, P5218
Poole, T. S., et al. 2008, MNRAS, 383, 627

Roming, P. W. A., et al. 2005, Space Sci. Rev., 120, 95

Scarpa, R., Urry, C. M., Falomo, R., Pesce, J. E., \& Treves, A. 2000, ApJ, 532, 740

Schlegel, D. J., Finkbeiner, D. P., \& Davis, M. 1998, ApJ, 500, 525

Stecker, F. W., Baring, M. G., \& Summerlin, E. J. 2007, ApJ, 667, L29

Tody, D. 1986, Proc. SPIE, 627, 733

Tody, D. 1993, in ASP Conf. Ser. 52, Astronomical Data Analysis Software and Systems II, ed. R. J. Hanisch, R. J. V. Brissenden, \& J. Barnes (San Francisco, CA: ASP), 173

Weekes, T. C., et al. 2002, Astropart. Phys., 17, 221

Wood, K. S., et al. 1984, ApJS, 56, 507

Zacharias, N., Monet, D. G., Levine, S. E., Urban, S. E., Gaume, R., \& Wycoff, G. L. 2004, BAAS, 36, 1418 